\title{
Design and test of robotic harvesting system for cherry tomato
}

\author{
Qingchun Feng ${ }^{1,2,3}$, Wei Zou ${ }^{1,2,3}$, Pengfei Fan ${ }^{1,2,3}$, Chunfeng Zhang ${ }^{1,2,3}$, Xiu Wang ${ }^{1,2,3 *}$ \\ (1. Beijing Research Center of Intelligent Equipment for Agriculture, Beijing Academy of Agriculture and Forestry Sciences, \\ Beijing 100097, China; 2. National Research Center of Intelligent Equipment for Agriculture, Beijing 100097, China; \\ 3. Beijing Key Laboratory of Intelligent Equipment Technology for Agriculture, Beijing 100097, China)
}

\begin{abstract}
Harvesting of fresh-eating cherry tomato was highly costly on labor and time. In order to achieve mechanical harvesting for the fresh-eating tomato, a new harvesting robot was designed, which consisted of a stereo visual unit, an end-effector, manipulator, a fruit collector, and a railed vehicle. The robot configuration and workflow design focused on the special cultivating condition. Three key parts were introduced in detail: a railroad vehicle capably moving on both ground and rail was adopted as the robot's carrier, a visual servo unit was used to identify and locate the mature fruits bunch, and the end-effector to hold and separate the fruit bunch was designed based on the stalk's mechanical features. The field test of the new developed robot was conducted and the results were analyzed. The successful harvest rate of the robot was $83 \%$, however, each successful harvest averagely needed 1.4 times attempt, and a single successful harvesting cycle cost 8 s excluding the time cost on moving.
\end{abstract}

Keywords: cherry tomato, harvesting robot, visual servo, configuration design, field test

DOI: $10.25165 /$ j.ijabe.20181101.2853

Citation: Feng Q C, Zou W, Fan P F, Zhang C F, Wang X. Design and test of robotic harvesting system for cherry tomato. Int J Agric \& Biol Eng, 2018; 11(1): 96-100.

\section{Introduction}

Fresh-eating tomato is greatly planted and consumed every year in China. More than $732000 \mathrm{hm}^{2}$ tomato is cultivated for fresh-eating ${ }^{[1]}$, and the annual consumption is $21 \mathrm{~kg}$ per person ${ }^{[1]}$. Timely harvesting is necessary to ensure the edible quality, however the harvesting cost is significantly increasing these years due to continuous rise of labor cost, and actually the harvesting cost has reached $1 / 4$ of the total $\operatorname{cost}^{[2]}$. Faced with agricultural population loss and labor cost increase, the automatic harvesting technology has caused extensive concerns for the potential application in the future.

As the typical application of robotic technology in agriculture ${ }^{[3,4]}$, harvesting robot has been researched since 1990s, and the prototypes for picking cucumber ${ }^{[5,6]}$, strawberry ${ }^{[7-11]}$, orange $^{[12]}$ have been developed. However, due to the especial characteristics in agricultural condition, such as unstable sunlight, unstructured work space, and soft mature fruits, it is more difficult for robots to automatically identify and operate the target in greenhouse than in other industry condition. So the robotic harvesting research still needs to overcome many challenges to achieve the commercial usage. The research on harvesting robot mainly focuses on three key points, including the target's identifying under complex background ${ }^{[13-15]}$, the soft fruit's reliable

\section{Received date: 2016-09-14 Accepted date: 2017-05-17}

Biograpgies: Qingchun Feng, $\mathrm{PhD}$ candidate, Senior Engineer, research interests: agricultural robot, Email: fengqc@nercita.org.cn; Wei Zou, Master, Engineer, research interests: electrical control, Email: zouw@nercita.org.cn; Pengfei Fan, PhD candidate, Engineer, research interests: mechanical engineering, Email: fanpf@ nercita.org.cn; Chunfeng Zhang, Master, Engineer, research interest: mechanical engineering, Email: zhangcf@nercita.org.cn.

*Corresponding author: Xiu Wang, $\mathrm{PhD}$, Professor, research interests: agricultural machine engineering. No.11, Shuguang Huayuan Middle Road, Haidian District, Beijing 100097, China. Tel: +86-10-51503346, Email: wangx@nercita.org.cn. separating ${ }^{[16,17]}$, and the configuration design fitting with the unstructured work space ${ }^{[18]}$. And an inexpensive, efficient, and adaptable solution for robotic harvesting is supposed as an acceptable result in future.

In this paper, a harvesting robot system for cherry tomato, the main fresh-eating tomato in China, was introduced, which is supposed to achieve the automatic pick for bunch-shaped fruit. In order to improve the robot's adaptability and reliability, the main design innovation was focused on visual unit and robot configuration. Besides, the robot's performance was tested in the greenhouse.

\section{Work condition for robotic harvesting}

The work condition for tomato harvesting robot is shown in Figure 1a. The distance between the plant rows is $1200 \mathrm{~mm}$. Through releasing the wire for hanging the plant, the mature tomato bunches for harvesting mainly existed in the space of $600-1200 \mathrm{~mm}$ above the ground, and 550-750 $\mathrm{mm}$ away from the row center. According to cultivating management for the longevity of tomato, the bottom leaves of plant will be removed manually during the maturing of fruits so that the fruits could absorb enough nutrients and sunlight, which is beneficial for automatic harvesting without the collision and overlapping of leaves. The harvesting robot is supposed to move on the rail in the center of the row space. As shown in Figure 1b, the tomato bunch is averagely $200 \mathrm{~mm}$ long, $70 \mathrm{~mm}$ wide, and its stalk length is about $30 \mathrm{~mm}$. Considering high labor cost of selectively harvesting the single fruit from the bunch, if $90 \%$ fruits of one bunch get mature, picking whole bunch other than a single fruit are both acceptable for manual and robotic harvesting, which would be easier and more efficient.

\section{Robotic harvesting method}

\subsection{Prototype model}

Based on the analysis on the work condition, the following 


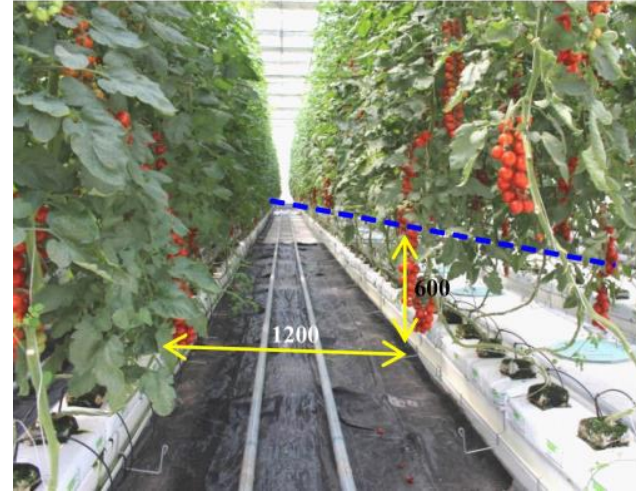

a. Cherry tomato cultivation

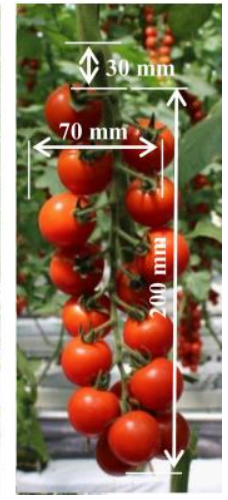

b. Tomato bunch
Figure 1 Work condition

design specifications are defined. The harvesting robot should be able to move autonomously through the greenhouse. It should be able to detect and locate the mature tomato bunches in the harvest region. Its operating space should be fit with the harvesting region so that all the fruits could be reached. The end-effector should reliably cut and handle the fruits to separate from the plant. Finally, the harvested fruits should be put into the fruit collector timely.

The harvesting robot model is shown in Figure 2. It consists of an autonomous vehicle, a 6-DOF manipulator, an end-effector, a stereo vision unit, a fruit collector and its controller. The view field center of the camera fixed on the manipulator coincides with manipulator's operating-space center, so that the robot's end-effector could reach as much region in its view field as possible. The fruit collector, composed by a container and a belt, is adapted to transfer the harvested fruits released from the end-effector to the container, so that the robot could have more time to locate and pick the fruit, instead of collecting fruits, which is supposed to decrease the harvesting time cost. The manipulator and collector are installed on an automatic elevator so that they could get fruits at different heights; besides, they could also be adjusted horizontally to fit with different row spaces. Each key part module will be described hereafter in detail.

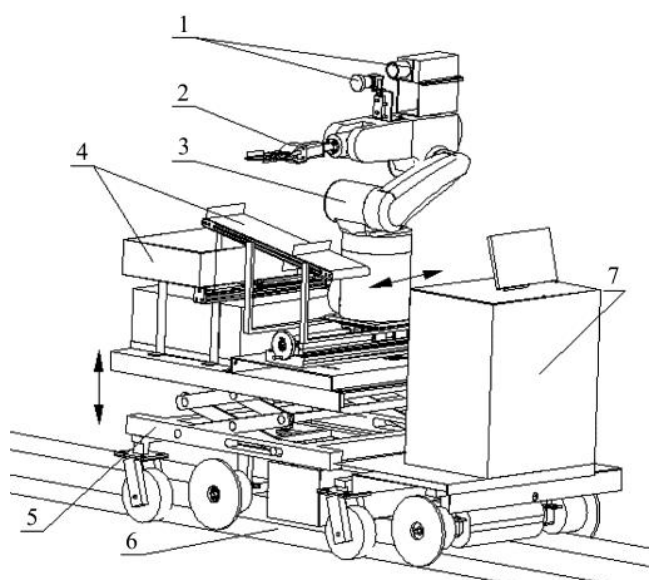

1. Stereo visual unit 2. End-effector 3. Manipulator 4. Fruit collector 5. Vehicle 6. Rail 7. Controller

Figure 2 Tomato harvesting robot configuration

\subsection{Workflow}

The harvesting robot moves ahead on the rails after initial setting. Once the vision unit identifies the mature fruit bunch based on the dynamic image information, the robot will stop moving and locate the bunch's 3-D position, and its manipulator operates the end-effector to approach the target bunch. After being picked from the plant, the fruit bunch is released to the transport belt, and transported to the fruit container. At the same time the manipulator resets its posture and begins to locate the mature bunch again. So far, the whole automatic harvesting cycle is finished. When all the mature bunches in robot's view field are harvested, manipulator will be raised by $300 \mathrm{~mm}$ to harvest at another height. And the robot will not move ahead again until all the mature fruits in the whole height range are picked. Finally, when the robot reaches the end of the plant row, a container with harvested fruit will be replaced by an empty one.

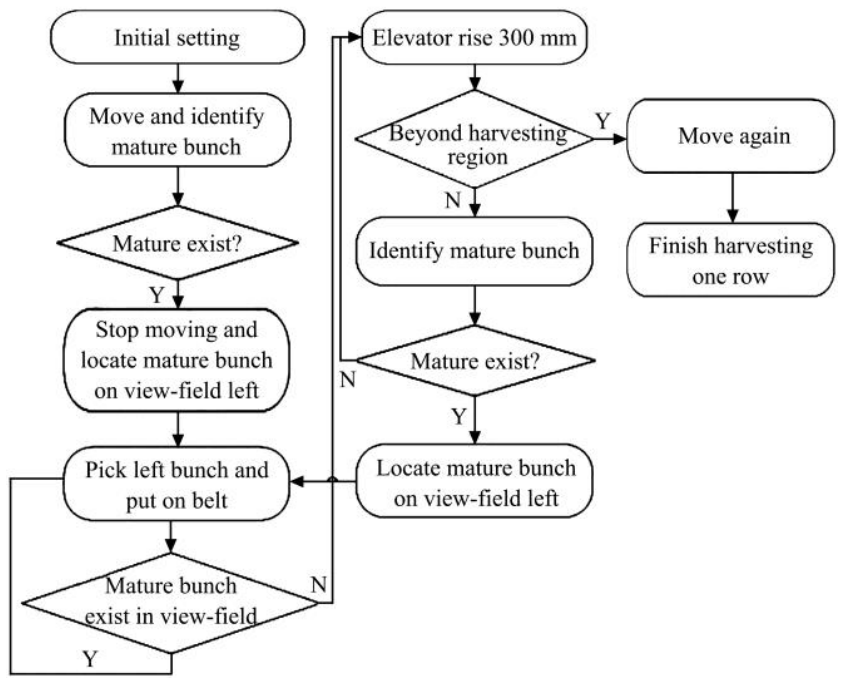

Figure 3 Robotic harvesting workflow

\section{Design of key parts}

\subsection{Railed vehicle}

As the robot's carrier moving on the rail in the row space when harvesting, the railed vehicle also needs to move on the ground to transfer from one row to another. Its wheel structure is shown in Figure 4. The rails are fixed on the ground surface of row end, and supported above the ground surface of inter-row. When railed wheels moves on rails, the universal wheels are suspensory as shown in right of Figure 4. And when the vehicle arrives at the row end, the universal wheels in front of railed wheel contact with ground surface, and raise the railed wheel above the rail as shown in left of Figure 4. Supported by the universal wheels, the robot could be transferred by human to another plant row on the hard-surface ground at the end of plant row, which spend about $1 \mathrm{~min}$. And an automatic winding machine is adopted to collect and release the electrical wire of the robot, through detecting the pull force on the wire, during the robot moving in the plant row.

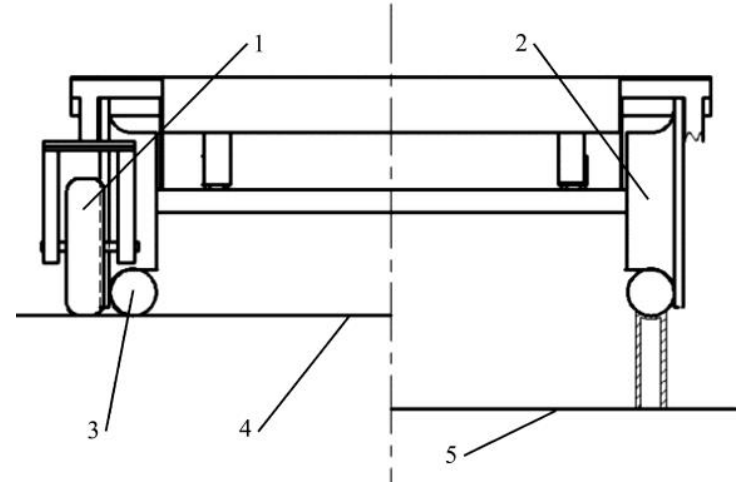

1. Universal wheels 2. Railed wheel 3. Rail 4. Ground surface of row end 5. Ground surface of row interior

Figure 4 Wheel structure of vehicle 


\subsection{Vision unit}

Accurately identifying and locating the mature fruit bunches is a key technique of harvesting robot research. In the previously published paper ${ }^{[14]}$, a new vision unit for cherry tomato's automatic harvesting was introduced. The unit includes a camera, a laser sensor and a manipulator, and its principle model is shown as Figure 5.

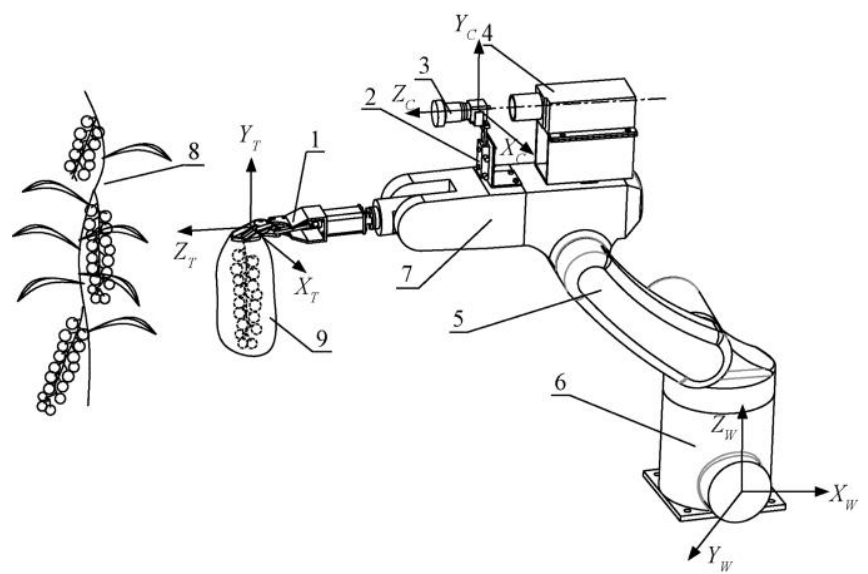

1. Grasper 2. Cylinder 3. Camera 4. Laser sensor 5. Manipulator 6. Base 7. Forearm 8. Plant 9. Fruit container

Note: $O-X_{W} Y_{W} Z_{W}$ represents world coordinate, $O-X_{C} Y_{C} Z_{C}$ represents camera coordinate and $O-X_{T} Y_{T} Z_{T}$ represents tool coordinate.

Figure 5 Fruit's targeting vision unit

\subsubsection{Mature bunch identification}

Through analyzing the color feature of the image acquired from the camera (Figure 6a), the R-G color model is adopted to intensify the difference between the target fruit and background. According to the column pixels grey statistics, the candidate area of fruit bunch is selected from the R-G image (Figure 6b). Then the CogPMAlignTool contained in the Cognex VisionPro image processing classlib is used for the fruits' identifying from the bunch (Figure $6 \mathrm{c})$, with the single fruit template of scaling $(0.8,1.2)$, rotating $(-\pi, \pi)$ and accepted score 0.36 .

\subsubsection{3-D position location}

According to the deviation between the top fruit's center and image center, the base joint and forearm joint of manipulator are controlled to change the posture of camera base on vision servo algorithm so that the two centers in image coincide approximately. After aiming the fruit, the laser sensor is triggered to measure the distance $D_{\text {laser }}$ between the vision system and the fruit. As the Equation (1), the accurate coordinate $\left(X_{w i}, Y_{w i}, Z_{w i}\right)$ of the periphery fruits could be obtained on the basis of the distance $D_{\text {laser }}$, the transition matrix $X^{-1}$ between the camera and the manipulator and the joint angels $\left\{\theta_{1}, \theta_{2} \ldots, \theta_{6}\right\}$ of manipulator.

$$
P_{w i}=\left[\begin{array}{c}
X_{w i} \\
Y_{w i} \\
Z_{w i} \\
1
\end{array}\right]=\left\{\theta_{1}, \theta_{2} \ldots \theta_{6}\right\} X^{-1}\left[\begin{array}{c}
0 \\
0 \\
D_{\text {Laser }} \\
1
\end{array}\right]
$$

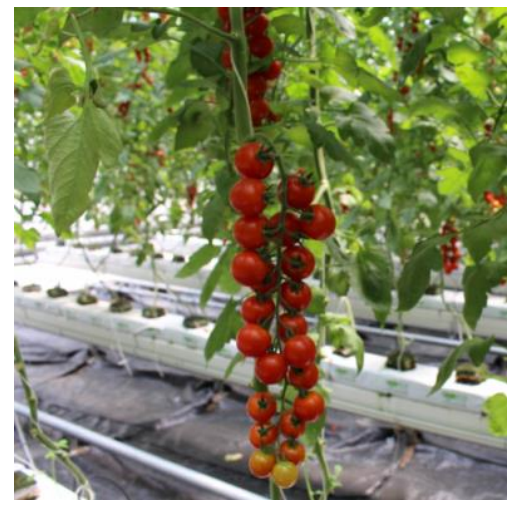

a. Original image

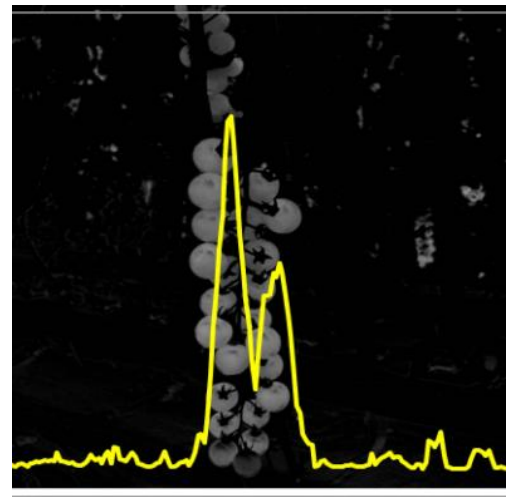

b. R-G image

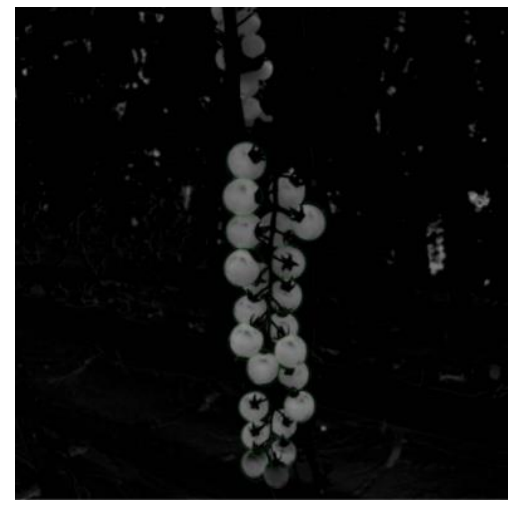

c. Fruits' recognizing result

Figure 6 Mature bunch identification

\subsection{Picking end-effector}

4.3.1 Stalk mechanical feature measurement

In order to improve the design accuracy of picking end-effector, the mechanical features of tomato stalk were measured, including frictional coefficient and cutting force. The measuring methods and results were introduced particularly in [18]. The static friction coefficient $f_{S}$ between stalk and rubber is 0.48 . The maximal cutting force with double blade is $17.52 \mathrm{~N}$. And the weight $G$ of tomato bunch is less than $0.8 \mathrm{~kg}$. If the end-effector could reliably hold and cut the tomato stalk, the pressure $F_{N}$ on stalk should satisfy the following Equation (2):

$$
\left\{\begin{array}{l}
G \leq F_{S} * F_{N} \\
F_{G} \leq F_{N}
\end{array}\right.
$$

\subsubsection{Design of end-effector}

The end-effector model is shown in Figure 7. The double cutter is used to cut the stalk, and the graspers fixed with cutters close or open to hold or release the stalk. The driving cylinder pull (or push) the active cutter to rotate. According to the mechanical feature measurement, the SMC CD55B20-30 cylinder is selected and the driving air pressure should be more than $0.3 \mathrm{MPa}$.

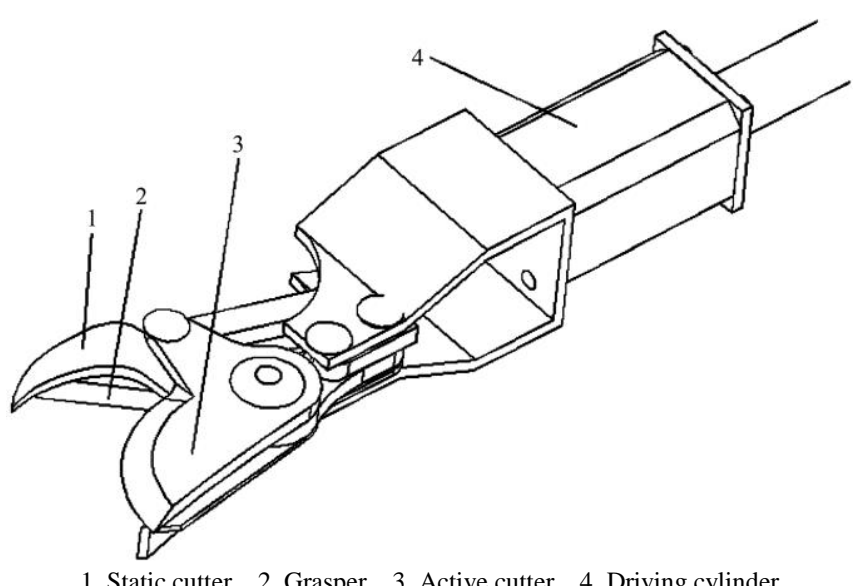

Figure 7 Picking end-effector 


\section{Test and analysis}

\subsection{Field test}

A field test was implemented in greenhouse of Beijing Special Vegetable Grand, 9:00-11:00, on December 18, 2015. Thirty bunches of tomato in 3 plant rows were selected as the test objects. Because all the leaves in harvesting region had been removed, which was the normal management for longevity tomato, the case of overlapped fruit bunch was ignored.

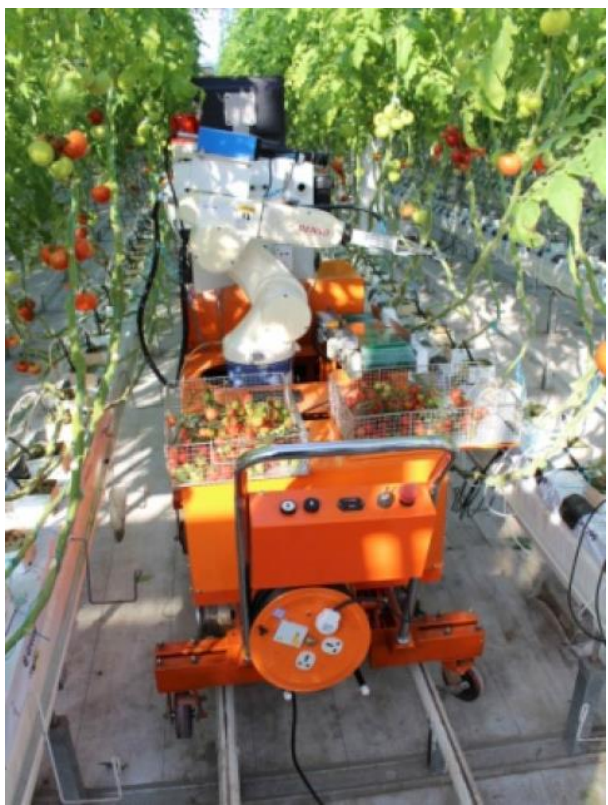

Figure 8 Field test picture

\subsection{Test results}

\subsubsection{Successful harvesting rate}

Among the 30 mature tomato bunches, 25 ones were successfully harvested, so the successful rate was about $83 \%$. However, the robot attempted for 42 times to harvest 30 bunches tomato, which means every successful harvest averagely need 1.4 times attempt because of serial operating mistakes. The harvesting attempt times was shown in Table 1. Among successfully harvested bunches, 18 ones were attempted for once, 6 ones for twice, and 4 ones for three times.

Table 1 Harvesting attempt results

\begin{tabular}{ccc}
\hline Attempt times & Bunches quantity & Successful harvesting times \\
\hline 1 & 18 & 18 \\
2 & 6 & 5 \\
3 & 4 & 2 \\
Total attempt times & 42 & 25 \\
\hline
\end{tabular}

The serial causes of the 12 times failed attempt were shown as Table 2. The failed attempt of 7 times was caused from end-effector's unreliable holding. Therefore the improvement on the end-effector is urgent to make it more compact and forceful so that less collision with plant stem happens and the bunch stalk can be grasped more strongly. Failed attempt because of the robot's limited workspace happened 3 times. When a mature bunch at the edge of view field was located by robot after it stopped to harvest a bunch in its view field center, the marginal bunch would be more likely out of the robot's workspace. However, this kind of mistake didn't directly cause the bunch's failed harvesting, because the bunch would move near to the view field center when robot stepped ahead. The mistake that the mature bunch was not found after robot stopped to identify happened 2 times, and the bunch would miss being harvested.

Table 2 Mistake attempt

\begin{tabular}{clc}
\hline No. & \multicolumn{1}{c}{ Description of mistake cause } & Times \\
\hline 1 & $\begin{array}{l}\text { The mature bunch existed out of the robot's workspace. } \\
2\end{array}$ & $\begin{array}{l}\text { End-effector failed to hold the stalk because of the collision } \\
\text { with main stem near the fruit bunch. }\end{array}$ \\
3 & $\begin{array}{l}\text { Mature bunch was not found by vision unit when robot } \\
\text { stopped to identify it. }\end{array}$ & 2 \\
4 & $\begin{array}{l}\text { Tomato stalk was not holding reliably, and fruit bunch fell } \\
\text { after being separated. }\end{array}$ & 2 \\
& Total number & 12 \\
\hline
\end{tabular}

5.2.2 Harvesting efficiency

The field test lasted over 25 min including robot moving along rail, and all the harvesting attempt of 42 times spent $10.3 \mathrm{~min}$ excluding robot moving, and a single successful harvesting cycle cost $8 \mathrm{~s}$, and the time cost of every task process was shown in Table 3. Except time cost of robot moving, the visual unit to identify and aim the target bunch cost the most time.

Table 3 Time cost composition

\begin{tabular}{ccc}
\hline No. & Working procedure & Time cost/s \\
\hline 1 & Find mature bunch, vehicle stop & 1 \\
2 & Identify and locate target bunch & 3 \\
3 & Manipulator approach target & 1.5 \\
4 & End-effector hold and cut & 0.5 \\
5 & Put bunch harvested onto belt & 1.5 \\
6 & Manipulator move to initial posture & 0.5 \\
& Total time cost & 8 \\
\hline
\end{tabular}

\subsection{Results analysis}

The failed harvest attempt was mainly caused from the collision between end-effector and plant stem, which meant it was not enough only to get the target position information in the unstructured greenhouse condition, but the 3-D information of plant stem and fruit stalk's posture were also needed. So a collision-free motion planning of the manipulator is urgent to research in the future. Besides, in order to successfully harvest the fruit bunch at the edge of robot's view field, a more intelligent vehicle could be useful, which is supposed to assist the vision unit to aim the target through its moving ahead or back so that the manipulator's work space could totally cover the harvest region.

As described in section 5.2.2, the robot's moving cost too much time, although slow moving is beneficial for identifying the mature fruit. Besides, the robot needed spend 3/8 time cost on aiming the target, so a more efficient algorithm is necessary to improve robot's efficiency. However, the commercial manipulator of DENSO VS-6556G is acceptable on accuracy and efficiency, and the fruit collector is also useful for manipulator to shorten the distance to release the fruit harvested and to reduce time cost of the single harvest cycle.

About the practical benefit and cost of the robotic harvester application, the performance of the robot is still not acceptable for commercial usage. It is supposed to be used only in the large-scale greenhouse where there is enough space for its moving at the plant row end. However, the prototype machine could intelligently work in greenhouse as expected, based on the present innovation on some key technology. And the future research would mainly focus on improving its efficiency and reliability until it performs as a commercial product. 


\section{Conclusions}

A new robot for harvesting cherry tomato bunch in particular cultivating mode was introduced in this paper. As a functional model, the robot could automatically move on the rail, identify and locate the mature bunch, hold and separate the target, even collect the fruit harvested. The field test results showed that the robot could successfully harvest $83 \%$ of the mature bunch prepared for test, although every successful harvest averagely need 1.4 times attempt. And a single successful harvesting cycle cost $8 \mathrm{~s}$ excluding time on moving. The future work on the robot will focus on conflict-free end-effector and rapid-aim vision unit.

\section{Acknowledgment}

We acknowledge that this work was financially supported by the National Natural Science Foundation of China (61703048), Beijing Excellent Talent Training to Support Young Key Individual Projects (2015000020060G134), and BAAFS Youth Research Fund (QNJJ201722).

\section{[References]}

[1] Zhang J. Analysis on international competitiveness of Chinese tomato industry. Wuxi: Jiangnan University, 2009. (in Chinese)

[2] Zhang $\mathrm{T}$, Wu W. Start with planning Chinese tomato industry. Academic Periodical of Farm Products Processing, 2009; 175(6): 108-114. (in Chinese)

[3] Zhang T, Yang L, Chen B, Zhang B. Research process of agricultural robot technology. Science China, 2010; 40(Supp.): 71-87. (in Chinese)

[4] Li P, Lee S, Hsu H. Review on fruit harvesting method for potential use of automatic fruit harvesting systems. Procedia Engineering, 2011; 23: 351-366.

[5] Henten E J V, Tuij B A J V, Hemming J, Kornet J G, Bontsema J, Os E A V. Field test of an autonomous cucumber picking robot. Biosystems Engineering, 2003, 86(3): 305-313.

[6] Ji C, Feng Q, Yuan T, Li W. Development and performance analysis on cucumber harvesting robot system in greenhouse. Robot, 2011; 33(6):
726-730. (in Chinese)

[7] Tarrio P, Bernardos A M, Casar J R, Besada J A. A harvesting robot for small fruit in bunches based on 3-D stereoscopic vision. Computers in Agriculture and Natural Resources, 4th World Congress Conference, Florida, 2006; pp.270-275.

[8] Feng Q, Wang X, Zheng W. A new strawberry harvesting robot for elevated-trough culture. Int J Agric \& Biol Eng, 2012; 5(2): 1-8.

[9] Kondo N, Ninomiya K, Hayashi S, Ota T, Kubota K. A new challenge of robot for harvesting strawberry grown on table top culture. ASAE Annual International Meeting, 2005; Paper Number: 053138.

[10] Shigehiko H, Kenta S, Satoshi Y, Ken K, Yasushi K, Junzo K, et al Evaluation of a strawberry-harvesting robot in a field test. Biosystems Engineering, 2010; 105: 160-171.

[11] Shiigi T, Mitsutaka K, Kondo N, Ninomiya K, Rajendra P, Kamata J, et al Strawberry harvesting robot for fruits grown on table top culture. ASABE Annual International Meeting, 2008; Paper Number: 084046.

[12] Muscato G, Prestifilippo M, Nunzio A. A prototype of an orange picking robot: past history, the new robot and experimental results. Industrial Robot, 2005; 32(2): 128-138.

[13] Feng Q, Cheng W, Zhou J, Wang X. Design of structured-light vision system for tomato harvesting robot. Int J Agric \& Biol Eng, 2014; 7(2): 19-26.

[14] Feng Q, Zhao C, Wang X, Wang X, Gong L, Liu C. Fruit bunch measurement method for cherry tomato based on visual servo. Transactions of the CSAE, 2015; 31(16): 206-212. (in Chinese)

[15] Tarrio P, Bernardos A M, Casar J R, Besada J A. A harvesting robot for small fruit in bunches based on 3-D stereoscopic vision. ASABE, 24 July 2006; Publication Number: 701P0606.

[16] Kondo N, Yata K, Iida M, Shiigi T, Monta M, Kurita M, et al Development of an end-effector for a tomato cluster harvesting robot Engineering in Agriculture, Environment and Food, 2010; 3(1): 20-24.

[17] Zhang K, Yang L, Zhang T. Design of an End-effector for strawberry harvesting robot. Journal of Agricultural Mechanization Research, 2009; 4: 54-56. (in Chinese)

[18] Feng Q, Ji C, Zhang J, Li W. Optimization design and kinematic analysis of cucumber harvesting robot manipulator. Transactions of the CSAM, 2010; 41(Supp): 244-248. (in Chinese)

[19] Li Z, Liu J, Li P. Relationship between mechanical property and damage of tomato during robot harvesting. Transactions of the CSAE, 2010; 26(5): 112-116. (in Chinese) 